\title{
Rolling Bearing Fault Diagnosis under Variable Conditions Using Hilbert-Huang Transform and Singular Value Decomposition
}

\author{
Hongmei Liu, ${ }^{1}$ Xuan Wang, ${ }^{1}$ and Chen Lu ${ }^{1,2}$ \\ ${ }^{1}$ School of Reliability and Systems Engineering, Beihang University, Beijing 100191, China \\ ${ }^{2}$ Science \& Technology on Reliability \& Environmental Engineering Laboratory, Beijing 100191, China \\ Correspondence should be addressed to Chen Lu; luchen@buaa.edu.cn
}

Received 11 April 2014; Revised 8 June 2014; Accepted 25 June 2014; Published 16 July 2014

Academic Editor: Pak-Kin Wong

Copyright (c) 2014 Hongmei Liu et al. This is an open access article distributed under the Creative Commons Attribution License, which permits unrestricted use, distribution, and reproduction in any medium, provided the original work is properly cited.

Fault diagnosis precision for rolling bearings under variable conditions has always been unsatisfactory. To solve this problem, a fault diagnosis method combining Hilbert-Huang transform (HHT), singular value decomposition (SVD), and Elman neural network is proposed in this paper. The method includes three steps. First, instantaneous amplitude matrices were obtained by using HHT from rolling bearing signals. Second, the singular value vector was acquired by applying SVD to the instantaneous amplitude matrices, thus reducing the dimension of the instantaneous amplitude matrix and obtaining the fault feature insensitive to working condition variation. Finally, an Elman neural network was applied to the rolling bearing fault diagnosis under variable working conditions according to the extracted feature vector. The experimental results show that the proposed method can effectively classify rolling bearing fault modes with high precision under different operating conditions. Moreover, the performance of the proposed HHTSVD-Elman method has an advantage over that of EMD-SVD or WPT-PCA for feature extraction and Support Vector Machine (SVM) or Extreme Learning Machine (ELM) for classification.

\section{Introduction}

In modern rapidly developing industries, the rolling bearing, a vital component of most rotary machines, faces an increasingly complex working environment. For the purpose of satisfying the production requirement, rolling bearing fault diagnosis is essential to ensure that rolling bearings work in a good state. In essence, rolling bearing fault diagnosis is approximately divided into several steps: signal processing to extract the feature vector, pattern recognition, and classification according to the extracted fault feature vector. Therefore, it is extremely important that the fault feature vector correctly and efficiently represents the fault characteristics hidden in the original signal, which also directly affects the classification results and diagnostic accuracy. In rolling bearing fault diagnosis, the variation of working conditions frequently leads to the change of most feature vital parameters [1] and even the entire diagnostic method. Adjusting the feature parameter or the diagnostic method according to the working condition is particularly labor intensive and time consuming.
Thus, it is essential to find a rolling bearing diagnostic method that is applicable to different working conditions.

Recently, researchers have paid more attention to the study of the rolling bearing fault diagnosis under variable working conditions. Based on a smart bearing, Shao presented a fault diagnosis system including several sensing devices to track the time-varying parameter [2]. Yang et al. introduced variable predictive model based class discrimination (VPMCD) into rolling bearing fault diagnosis [3]. In order to achieve the fault diagnosis under variable conditions, Yang et al. employed the feature vector obtained from different working conditions as the input of the classifier. $\mathrm{He}$ et al. addressed manifold learning on generated timefrequency distributions for machine fault signature analysis [4]. These previous studies have a pivotal function in fault diagnosis under variable conditions. However, some of these studies require additional sensing devices that increase the system cost; some conduct feature extraction under as many working conditions as possible to ensure the robustness; some decrease the algorithm robustness because of complex 
calculation. How to certify the robustness and low costing of the fault diagnosis under variable working conditions still needs further study.

At present, there exist many conventional methods employed to process the vibration signal: the Fourier transform, the short-time Fourier transform, the Wigner-Ville distribution, and the wavelet transform. When processing the signal, the Fourier transform is only applicable to linear system and stationary signal. Otherwise, the result will lose its physical meaning. However, the vibration signal of rollingbearings is characterized by nonstationarity, which makes the Fourier transform unsuitable for extracting the feature of the nonstationary signal. As time-frequency analysis methods, the short-time Fourier transform [5], the Wigner-Ville distribution [6], and the wavelet transform [7] can process the nonstationary signal. However, they all have respective limitation. Due to Heisenberg's uncertainty principle, the finest time location and the best frequency resolution cannot be reached simultaneously [8], which restricts the application of the short-time Fourier transform. Because of the presence of cross-term interference, the Wigner-Ville distribution is limited. Meanwhile, although the wavelet transform has demonstrated its superiority in processing the nonstationary signal, the energy leakage issue is inevitable. Besides, the base function needs a preselection according to the structure of the signal, and the optimization problem for base function preselection still requires a widely accepted resolution. By contrast, the Hilbert-Huang transform (HHT) [9] is free from the restriction of Heisenberg's uncertainty principle. Its essence is processing the signal with Empirical Mode Decomposition (EMD) [10] to get the intrinsic mode function (IMF) and applying the Hilbert transform to each IMF for Hilbert spectrum analysis. Based on its own time scale characteristics, the EMD method decomposes signals without any basis function preselection. In theory, the EMD method can be applied to any type of signal decomposition; therefore, it has an extremely distinct advantage in handling nonstationary and nonlinear data. It is able to decompose a complicated signal into a finite number of IMFs, and the decomposed IMFs contain the local characteristic of the original signal at different time scales.

Under variable working conditions, the rolling bearing fault characteristic frequency changes over time, thereby necessitating time-frequency analysis. Compared with other time-frequency methods, Hilbert-Huang transform (HHT) has a distinct advantage in handling nonstationary and nonlinear data. Consequently, HHT is implemented in this research for time-frequency analysis. Nevertheless, the result obtained from HHT under variable working conditions is tremendous and complex; thus, dimensionality reduction is required for the purpose of enhancing classification accuracy. In addition, it is extremely complicated to extract the corresponding amplitude at the time-varying characteristic frequency. In comparison, searching a fault feature insensitive to the working condition variation is preferred. Theoretically, the PCA and the singular value decomposition (SVD) can both be employed for dimensionality reduction. Moreover, the singular value has considerable stability [11] and changes negligibly when matrix elements change. Thus, the singular value can maintain satisfactory stability under variable working conditions, thereby enhancing the robustness of the fault diagnosis under variable conditions.

Thus, HHT in tandem with SVD is proposed for rolling bearing fault feature extraction under variable working conditions in this paper. To begin with, time-frequency analysis is implemented on the vibration signal by utilizing HHT, and the instantaneous amplitude and instantaneous frequency are obtained. Afterwards, for the purpose of dimensionality reduction and obtaining the feature insensitive to working condition variation, SVD is employed to the instantaneous amplitude to obtain the feature vector. After the feature vector is obtained, some intelligent classification techniques can be implemented for intelligent rolling bearing fault classification, such as Support Vector Machine (SVM) [12], Relevance Vector Machine (RVM) [13], and Extreme Learning Machine [14]. Similar to other binary classification algorithms, SVM cannot solve multiclassification efficiently. RVM is restricted by the long training time. As a feedforward network, ELM is characterized by fast learning and considerable generalization performance. However, the model accuracy of the feedforward network is lower than that of the feedback network. Consequently, Elman neural network, a typical feedback neural network, is employed for rolling bearing fault identification and classification in this paper.

This paper is organized as follows. Section 2 introduces the principle of the diagnostic method based on HHT and SVD. Section 3 presents fault identification and classification scheme. The experimental results are reported in Section 4. Conclusions are drawn in Section 5.

\section{Feature Extraction Method Based on HHT and SVD}

Fault diagnosis for rolling bearings is comprised of fault feature extraction and fault classification. Fault features extraction is the most important procedure during the fault diagnosis, and the fault is then classified according to the extracted feature.

2.1. Time-Frequency Signal Decomposition Based on HHT. As a self-adaptive data decomposition method, HHT is based on partial time scales and therefore can deal with both nonlinear and nonstationary process. HHT could transfer any complex signal into the linear superposition of several intrinsic mode function (IMF) components. In HHT, the analytic signal can be constructed by Hilbert transform, in advance; the instantaneous amplitude and instantaneous frequency can be obtained. Additionally, the Hilbert spectrum can be obtained after further processing.

HHT [10], which combines Empirical Mode Decomposition with Hilbert transform, can be applied to determine the instantaneous amplitude and the instantaneous frequency.

(1) Empirical Mode Decomposition. After the implementation of the EMD, the original signal $x(t)$ can be decomposed as follows:

$$
x(t)=\sum_{i=1}^{n} c_{i}(t)+r_{n}(t),
$$


where $c_{i}(t)$ is the $i$ th IMF and $r_{n}(t)$ is the residue. As the constant sequence or monotonic sequence, $r_{n}(t)$ represents the average trend of signal. The IMF component $c_{i}(i=1, \ldots, n)$ represents the signal from high frequency to low frequency in different frequency bands. The frequency component of the signal in each frequency band changes according to the original signal.

From the perspective of the basis function, the basis functions are different during the application of EMD. It differs from the Fourier transform (taking a series of sine waves or cosine waves with constant amplitude and frequency as the basis function) and the wavelet transform (taking the preset basis function). Consequently, EMD is beneficial to enhance the signal decomposition efficiency and to process the nonstationary data.

(2) Hilbert Transform. In essence, Hilbert transform is the convolution of signal with $1 / \pi t$, which makes it possible to calculate instantaneous parameters for transient signal and complex signal.

When the IMF is set as equal to $c(t)$, the analytic signal $h(t)$ of $c(t)$ is as follows:

$$
h(t)=c(t)+j \widetilde{c}(t)=a(t) e^{j \varphi(t)},
$$

where $\widetilde{c}(t)$ is the outcome function after applying Hilbert transform to $c(t)$ and is expressed as

$$
\widetilde{c}(t)=\frac{1}{\pi} \int_{-\infty}^{+\infty} \frac{c(t)}{t-\tau} d \tau .
$$

$a(t)$ is the amplitude function and the envelope signal of the IMF component $c(t)$ is expressed as

$$
a(t)=\sqrt{c^{2}(t)+\tilde{c}^{2}(t)} .
$$

$\varphi(t)$ is the phase function:

$$
\varphi(t)=\tan ^{-1}\left(\frac{\widetilde{c}(t)}{c(t)}\right) .
$$

We calculate the derivation of the phase function to obtain the instantaneous frequency, as follows:

$$
\omega(t)=\frac{d \varphi(t)}{d t} .
$$

Accordingly, the original signal $x(t)$ can be expressed by a time-dependent function containing instantaneous amplitude and instantaneous frequency, as follows:

$$
H(w, t)=\operatorname{Re} \sum_{i=1}^{n} a_{i}(t) \exp \left(j \int w_{i}(t) d t\right) .
$$

In this way, we are equipped with the ability to conduct the observation and analysis intuitively from the Hilbert spectrum, which is a three-dimensional plot with time axis, frequency axis, and amplitude axis. Because the Hilbert spectrum can provide the amplitude contribution at each frequency and each time [15], it is possible to handle the rolling bearing fault diagnosis under time-varying conditions by applying HHT.

The instantaneous amplitude of the IMF is its envelope signal, which is similar to the periodic signal with large amplitude, long duration, and unchangeable repetition rate, as well as no low-frequency interference [16]. The envelope signal is beneficial in identifying faults, even weak ones. Thus, we can calculate the amplitude of each analytic signal $h(t)$ to obtain $a(t)$ for further analysis. However, it is complicated to calculate the accurate fault characteristic frequency and to extract the corresponding amplitude from $a(t)$ under variable working conditions. Thus, rolling bearing fault identification is difficult under variable operating conditions. To address this issue, we further propose SVD in tandem with HHT for rolling bearing fault feature extraction.

2.2. SVD Based on HHT. SVD is a matrix transformation algorithm, which is defined in the following process. We assume that $M$ is a $m \times n$ matrix. In SVD, any matrix $M \epsilon$ $R^{m * n}$ can be decomposed into three matrices, which are $U \in$ $R^{m * m}, \sum \in R^{m * n}$, and $V \in R^{n * n}[8]$ as follows:

$$
M=U \sum V^{*},
$$

where $U$ and $V$ are orthogonal and $\sum$ is a diagonal matrix with $r=\operatorname{rank}(M)$ principal positive diagonal entries:

$$
\sum=\left(\begin{array}{ccccc}
\sigma_{1} & 0 & \cdots & 0 & 0 \\
0 & \sigma_{2} & \cdots & 0 & 0 \\
\vdots & \vdots & \cdots & \vdots & \vdots \\
0 & 0 & \cdots & \sigma_{r-1} & 0 \\
0 & 0 & \cdots & 0 & \sigma_{r}
\end{array}\right)
$$

$\sigma_{i}$ are considered as the singular values of $M$. The singular values are listed in descending order automatically by SVD function, as follows:

$$
\sigma_{1}(M) \geq \sigma_{2}(M) \geq \cdots \geq \sigma_{r}(M) .
$$

The singular value has considerable stability and changes negligibly when matrix elements change [17]. In this paper, SVD is used to determine the intrinsic characteristic of instantaneous amplitude matrices obtained from HHT. Singular values can express the feature of the original matrix in the form of several values, which is conducive to compressing the scale of the feature vector.

As shown in Figure 1, the original vibration signal is firstly decomposed by EMD, and IMFs are then obtained. The analytic signal $h_{i}$ is obtained after each major IMF is processed by the Hilbert transform (see formula (2)). Subsequently, the instantaneous amplitude $a_{i}$ is obtained after calculating the amplitude function of each $h_{i}$ (see formula (4)). The feature matrix $M$ is constructed by $a_{i}(i=1, \ldots, k)$. To a certain degree, the number of IMFs is decided by the characteristic, length, and complexity of the target signal. For the purpose of convenient analysis, for a signal with given length, normally the signal needs to be divided into a number of subsignals with fixed length. Generally, due to the difference in the subsignals, the numbers of obtained 


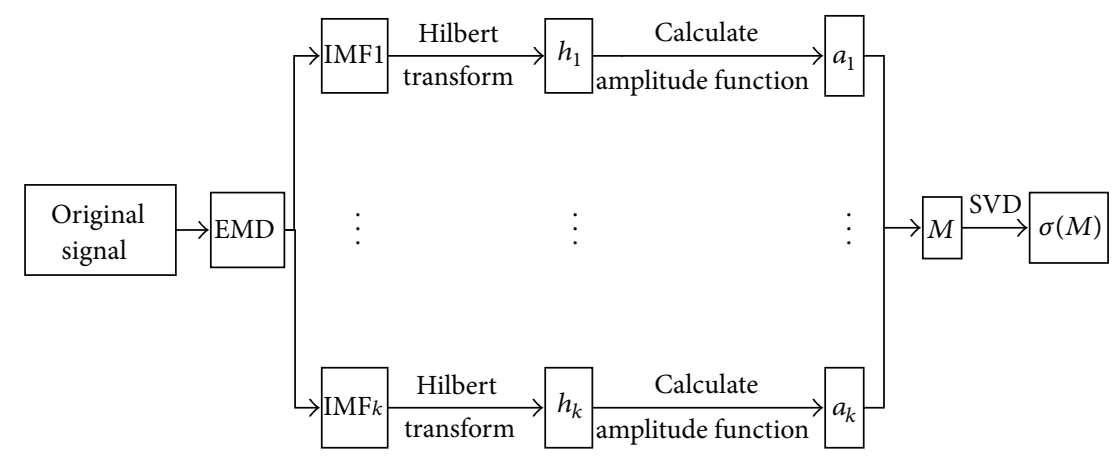

FIGURE 1: Flowchart of obtaining the feature vector.

IMFs will normally be different. To all subsignals, if the minimum number of obtained IMFs is $k 1$, the feature matrix's dimension $k$ should be no larger than $k 1$. In addition, the first few IMFs have the highest frequency and largest energy, while the last few IMFs keep relatively moderate. That is, the first few IMFs comprise most fault information. Consequently, the prior $k$ IMFs are then extracted for analysis. From the perspective of practice, $k$ is usually set as $3 \sim 5$. In this paper, according to the decomposition results of raw vibration signal, we set $k=5$, so the feature matrix $M$ can be expressed as follows:

$$
M=\left(\begin{array}{c}
a_{1} \\
a_{2} \\
\vdots \\
a_{k}
\end{array}\right)
$$

After the application of SVD to $M$, the singular value vector as well as the fault feature vector $\sigma(M)$ can be obtained as follows:

$$
\sigma(M)=\left[\sigma_{M}^{1}, \sigma_{M}^{2}, \ldots, \sigma_{M}^{k}\right] .
$$

The fault is then identified and classified according to the obtained fault feature vector $\sigma(M)$.

\section{Fault Identification and Classification Based on Elman Neural Network}

In intelligent fault diagnosis, neural networks are always used to classify the fault mode. In this study, the Elman neural network was used for fault classification.

The Elman neural network includes input, output, hidden, and context layers. When the output of the hidden layer neurons goes through the context layer, it associates with the input of the hidden layer [18]. This type of association is sensitive to the historical state of data; internal feedback increases the capacity of the network to deal with dynamic information, overcoming the drawback of the feed-forward network, which does not have dynamic characteristics. Therefore, compared with other neural networks, the Elman neural network is more applicable to classify the fault mode. The structure of the Elman neural network is shown in Figure 2.

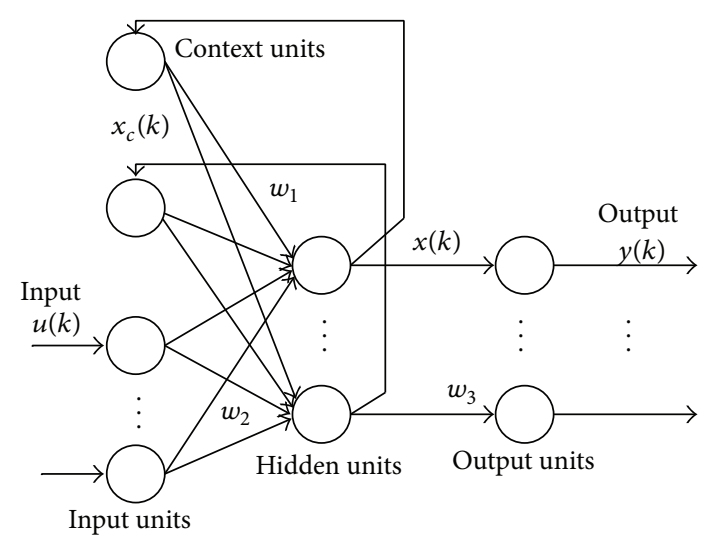

FIGURE 2: Elman neural network.

The transfer function of the hidden layer in the Elman neural network is a sigmoid function. The activation function of the output layer neuron is a linear function. The nonlinear state equations of the network are

$$
y(k)=g\left[w_{3} x(k)\right],
$$

where $y$ represents the output vector of the network, $x$ denotes the output vector of the hidden layer, $w_{3}$ is the connection matrix from the context layer to the output layer, and $g(\cdot)$ denotes the activity function of the output neurons. Consider the following:

$$
\begin{gathered}
x(k)=f\left[w_{1} x_{c}(k)+w_{2} u(k-1)\right], \\
x_{c}(k)=x(k-1) .
\end{gathered}
$$

$u$ is the input vector of the network, $w_{2}$ is the connection matrix from the input layer to the hidden layer, $w_{1}$ represents the linked weights from the context layer to the hidden layer, $x_{c}$ represents the feedback state vector, and $f(\cdot)$ represents the transfer function of the hidden layer.

\section{Experimental Result and Discussion}

To validate the effectiveness of the proposed fault diagnosis method, we used the vibration data from Bearing Data Center of Case Western Reserve University. The 6205-2RS deepgroove ball bearing is employed in the experiment. As shown 
TABLE 1: Relevant information for dataset.

\begin{tabular}{lccccc}
\hline & & \multicolumn{4}{c}{ Groups } \\
Label & Status & Working condition & \\
& & 0 HP (1797 RPM $)$ & 1 HP (1772 RPM) & 2 HP (1750 RPM) & 3 HP (1720 RPM) \\
\hline 1 & Normal & 24 & 24 & 24 & 24 \\
2 & Inner-race fault & 24 & 24 & 24 & 24 \\
3 & Outer-race fault & 24 & 24 & 24 & 24 \\
4 & Rolling element fault & 24 & 24 & 24 \\
\hline
\end{tabular}

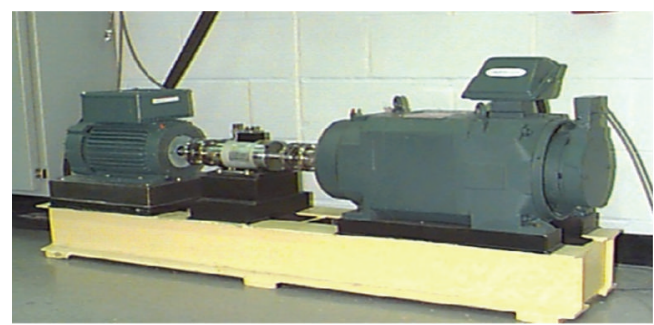

FIgURe 3: Test bed.

in Figure 3, the test bed consists of $1.5 \mathrm{~kW}$ motor, torque sensor/encoder, dynamometer, and electrical control devices. The motor drives the input shaft and the output shaft drives the load. The vibration data are sampled under different working conditions: for motor loads of 0 to 3 horsepower (HP) (motor speeds of 1797 to $1720 \mathrm{RPM}$ ). In addition, different faults such as inner-race fault, outer-race fault, and rolling element fault were injected in the experiment. The data of different fault states are acquired with the sampling frequency of $12 \mathrm{kHz}$.

4.1. Performance for Different Working Conditions. Before the implementation of SVD, the original vibration signal is processed by HHT to obtain the Hilbert spectrum and the instantaneous amplitude. The Hilbert spectrum of different states is shown in Figure 4(a) Figure 4(d). The Hilbert spectrum of the normal state in Figure 4(a) indicates that the energy distribution is uniform, whereas the peak appears in the frequency band at approximately $1,000 \mathrm{~Hz}$. The maximum amplitude is approximately 0.09. The Hilbert spectrum in Figure 4(b) indicates that the dominant energy distributes in the frequency band at approximately $3,000 \mathrm{~Hz}$, and the time-frequency distribution is complicated under the inner-race fault mode. The maximum amplitude is approximately 0.9 . For the outer race fault mode, the Hilbert spectrum in Figure 4(c) shows that the dominant energy distribution is similar to that of the innerrace fault mode. Nevertheless, the maximum amplitude is approximately 3 , which indicates that the vibration energy is relatively high. The Hilbert spectrum of the rolling element fault illustrates that energy rarely distributes in the frequency band from 2,000 Hz to 3,000 Hz. Moreover, in Figure 4(d), the vibration energy is low and its maximum amplitude is only approximately 0.35 . The time-frequency distribution varies under different states. Therefore, the feature matrix can be constructed for fault identification.

After obtaining the instantaneous amplitude matrices, we can obtain the singular value vector by conducting SVD.
As shown in Table 1, four datasets sampled under normal state, inner-race fault state, outer-race fault state, and rolling element fault state were obtained. Each dataset is divided into four subdatasets by different working conditions, and each subdataset contains 24 groups of data. Therefore, the whole four datasets contain 384 samples, and the feature extraction method based on HHT and SVD is implemented on each sample. For visualization, the scatter plots of the first three singular values extracted by HHT and SVD for datasets in Table 1 are shown in Figure 5. As shown in Figure 5, even under different working conditions, the singular value vectors extracted under the same failure mode retain a high degree of coincidence. Namely, the feature vector extracted by HHT in tandem with SVD is insensitive to the working condition variation. In addition, the singular value vectors corresponding to the three fault modes and the normal state have satisfactory mode separability. Due to the good separability of different fault states, the singular value can be feasibly used as the input of the neural network classifier.

4.2. Comparison between the Proposed Method with EMD and WPT in Tandem with PCA for Feature Extraction. In this subsection, another two widely applied methods, EMD and WPT (wavelet packet transform) in tandem with PCA, are also implemented on the same datasets in Table 1, and their performance is compared with that of HHT in tandem with SVD for rolling bearing feature extraction under variable working conditions.

To begin with, EMD in tandem with $\operatorname{SVD}[19,20]$ is exploited, SVD is applied to the first 5 IMFs, and then the feature vector is extracted. The scatter plots of the first three singular values extracted by EMD and SVD for datasets in Table 1 are shown in Figure 6. As shown in Figure 6, there exist major fluctuations for the corresponding feature vector, especially for inner-race fault and outer-race fault; that is, the feature vector obtained by EMD and SVD is affected greatly by working condition variation. The clustering interval between inner-race fault and outer-race fault is relatively small, which may lead to the misclassification. Consequently, the feature extraction method based on EMD and SVD is unable to work effectively and stably under variable working conditions.

Afterwards, another widely used method, WPT in tandem with PCA [21], is employed for feature extraction. The signal is decomposed by WPT into 3 levels, and then the signal can be decomposed into 8 frequency bands. The energy of each frequency band is then calculated to obtain wavelet packet energy feature. Subsequently, PCA is used to extract principal components from the wavelet packet 


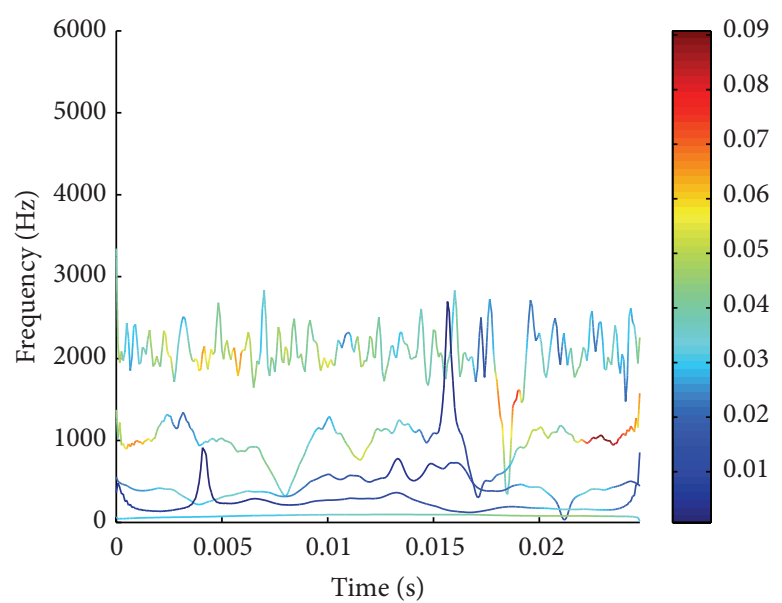

(a)

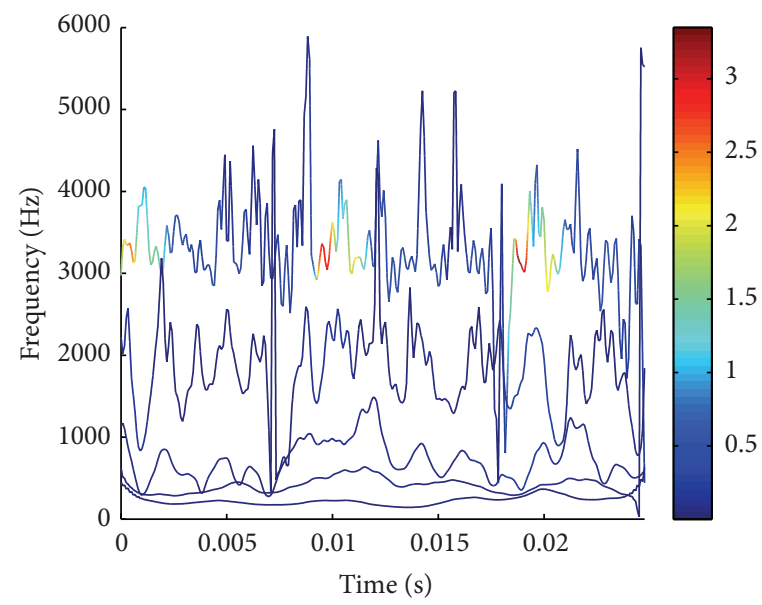

(c)

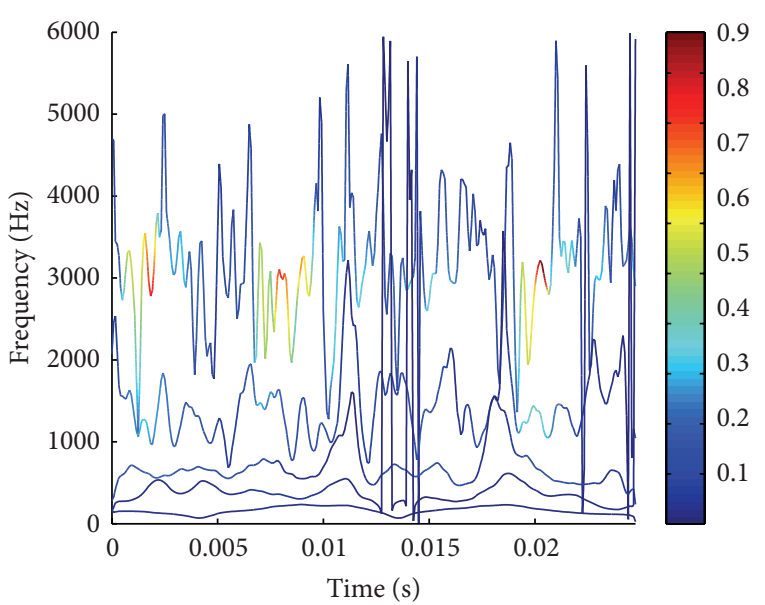

(b)

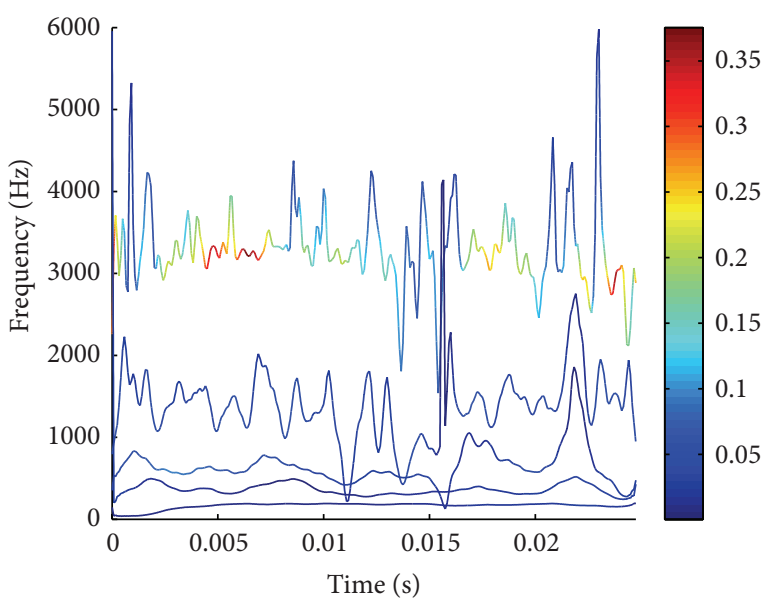

(d)

Figure 4: Hilbert spectra of (a) normal, (b) inner-race fault, (c) outer-race fault, and (d) rolling element fault.

energy feature for dimensionality reduction. The scatter plots of the first three PCs (principal components) extracted by WPT and PCA for datasets in Table 1 are illustrated in Figure 7. As shown in Figure 7, there exist major fluctuations for all states. Namely, the feature vector obtained by WPT and PCA is sensitive to the working condition variation. In addition, the clustering intervals between three fault states are relatively small, which may lead to the incorrect classification. Consequently, WPT in tandem with PCA is also unsuited for rolling bearing fault diagnosis under variable working conditions. By contrast, the feature vector extracted by HHT in tandem with SVD keeps considerable stability even under different working conditions, and the mode separability for different fault states is satisfactory. For rolling bearing feature extraction under variable working conditions, the proposed method performs better than EMD in tandem with SVD and WPT in tandem with PCA.

4.3. Classification Performance Using Elman Neural Network. Through the proposed feature extraction based on HHT and SVD, singular value vectors are obtained as the feature vectors. The faults status is then identified and classified by using the Elman neural network. The input of the Elman neural network is the singular value feature, and the output is set as $\left(\begin{array}{llll}1 & 0 & 0 & 0\end{array}\right),\left(\begin{array}{llll}0 & 1 & 0 & 0\end{array}\right),\left(\begin{array}{llll}0 & 0 & 1 & 0\end{array}\right)$, and $\left(\begin{array}{llll}0 & 0 & 0 & 1\end{array}\right)$, which represent normal, inner-race fault, outer-race fault, and rolling element fault in sequence. As shown in Table 2, the datasets are divided into a training dataset with 20 groups of data (the neural network training set is not listed in this paper because of space limitations) and a testing dataset with 364 groups of data. The partial results of network neural recognition are reported in Table 3. In addition, another two classification techniques, Support Vector Machine (SVM) and Extreme Learning Machine (ELM), are applied to the same training data and testing data in Table 2. Afterwards, the corresponding classification performance of Elman neural networks, SVM, and ELM is illustrated in Figure 8.

To verify the robustness of the proposed method, the Elman neural network is trained under a harsh situation: small training dataset (5 groups of data for each fault status) is used, and only data under a single working condition (3HP for this paper) is contained. Even so, the actual output 
TABLE 2: Training data and testing data statistics.

\begin{tabular}{|c|c|c|c|c|c|c|}
\hline \multirow{3}{*}{ Label } & \multirow{3}{*}{ Status } & \multicolumn{5}{|c|}{ Working condition } \\
\hline & & \multicolumn{4}{|c|}{ Testing data } & \multirow{2}{*}{$\begin{array}{c}\text { Training data } \\
3 \mathrm{HP}\end{array}$} \\
\hline & & $0 \mathrm{HP}$ & $1 \mathrm{HP}$ & $2 \mathrm{HP}$ & $3 \mathrm{HP}$ & \\
\hline 1 & Normal & 24 & 24 & 24 & 19 & 5 \\
\hline 2 & Inner-race fault & 24 & 24 & 24 & 19 & 5 \\
\hline 3 & Outer-race fault & 24 & 24 & 24 & 19 & 5 \\
\hline 4 & Rolling element fault & 24 & 24 & 24 & 19 & 5 \\
\hline
\end{tabular}

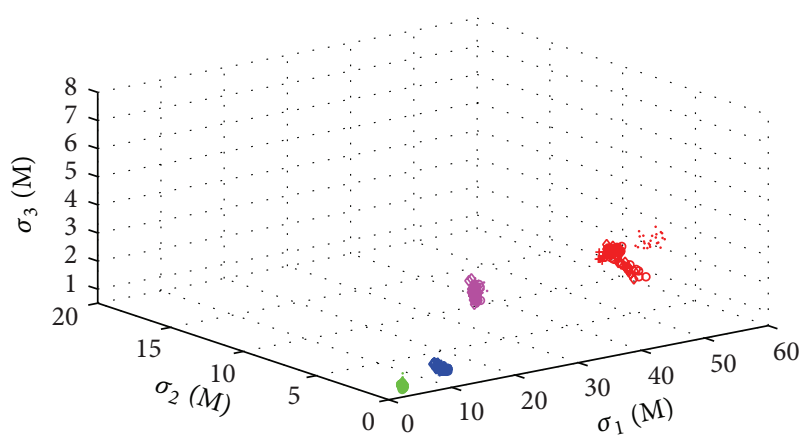

- Normal (0HP)

- Normal (1HP)

+ Normal (2HP)

- Normal (3HP)

- Inner-race fault (0HP)

- Inner-race fault (1HP)

+ Inner-race fault $(2 \mathrm{HP})$

- Inner-race fault (3HP)
- Outer-race fault (0HP)

- Outer-race fault (1HP)

+ Outer-race fault (2HP)

- Outer-race fault (3HP)

- Rolling element fault (0HP)

- Rolling element fault (1HP)

+ Rolling element fault (2HP)

- Rolling element fault (3HP)

Figure 5: Scatter plots of the first three singular values extracted by HHT-SVD for datasets in Table 1.

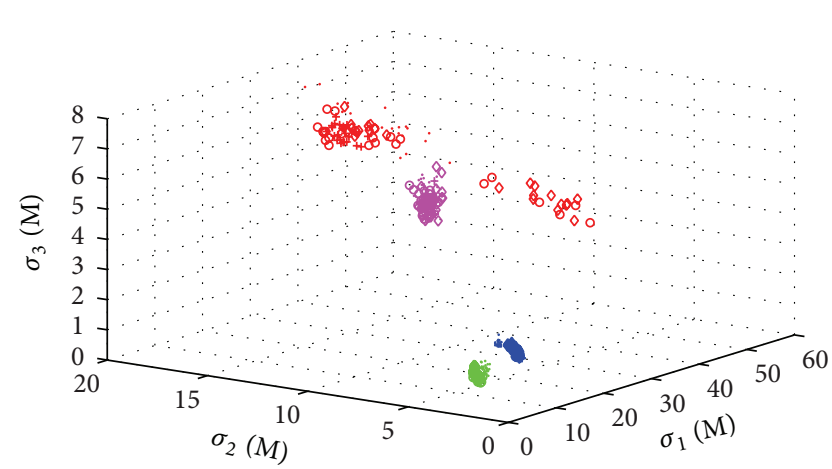

- Normal (0HP)

- Normal (1HP)

+ Normal (2HP)

$\checkmark$ Normal (3HP)

- Inner-race fault (0HP)

- Inner-race fault (1HP)

+ Inner-race fault (2HP)

- Inner-race fault (3HP)

FIGURE 6: Scatter plots of the first three singular values extracted by EMD-SVD for datasets in Table 1.

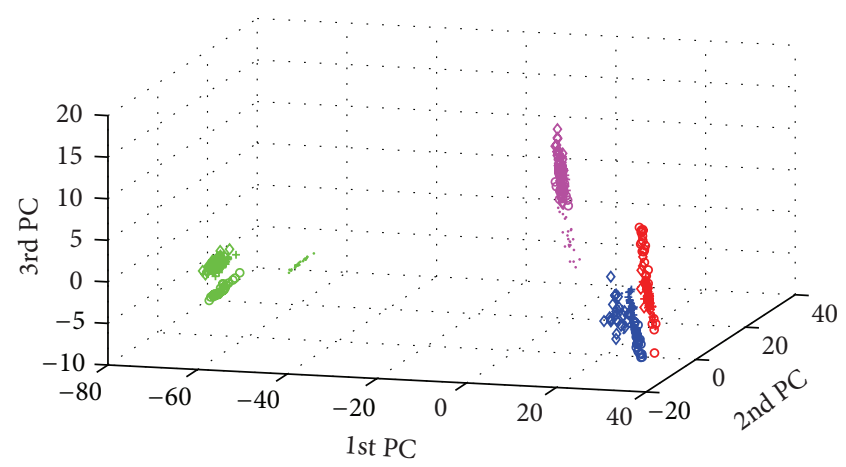

$$
\begin{array}{ll}
- \text { Normal (0HP) } & \cdot \text { Outer-race fault }(0 \mathrm{HP}) \\
\circ \text { Normal (1HP) } & \circ \text { Outer-race fault }(1 \mathrm{HP}) \\
+ \text { Normal }(2 \mathrm{HP}) & + \text { Outer-race fault }(2 \mathrm{HP}) \\
\diamond \text { Normal }(3 \mathrm{HP}) & \diamond \text { Outer-race fault }(3 \mathrm{HP}) \\
- \text { Inner-race fault }(0 \mathrm{HP}) & \cdot \text { Rolling element fault }(0 \mathrm{HP}) \\
\circ \text { Inner-race fault }(1 \mathrm{HP}) & \circ \text { Rolling element fault }(1 \mathrm{HP}) \\
+ \text { Inner-race fault }(2 \mathrm{HP}) & + \text { Rolling element fault }(2 \mathrm{HP}) \\
\diamond \text { Inner-race fault }(3 \mathrm{HP}) & \diamond \text { Rolling element fault }(3 \mathrm{HP})
\end{array}
$$

FIGURE 7: Scatter plots of the first three PCs extracted by WPT and PCA for datasets in Table 1.

of the neural network significantly agrees with the target output under variable conditions, as shown in Table 3. To a certain degree, parameters do not need to change under variable working conditions. As shown in Figure 8, the classification accuracy of Elman neural network is $100 \%$. In comparison, some samples of inner-race fault are classified incorrectly into the outer-race fault by SVM. The same misclassification follows in ELM. The classification accuracy of SVM and ELM is $97.5 \%$ and $98.6 \%$, respectively. The performance of Elman neural network outperforms that of SVM or ELM for classification. Consequently, the proposed method combining HHT-SVD with Elman neural network can be effectively used in rolling bearing fault diagnosis under variable working conditions.

4.4. Case Study for Rolling Bearing Fault Diagnosis under Time-Varying Conditions. To further verify the effectiveness of the rolling bearing fault diagnosis method proposed in this paper, this method is also used to detect the burst fault under the time-varying condition, which is exhibited in Figure 9. The time-varying data is acquired by connecting data segments under different working conditions and different fault modes. The initial state of the rolling bearing was normal, 

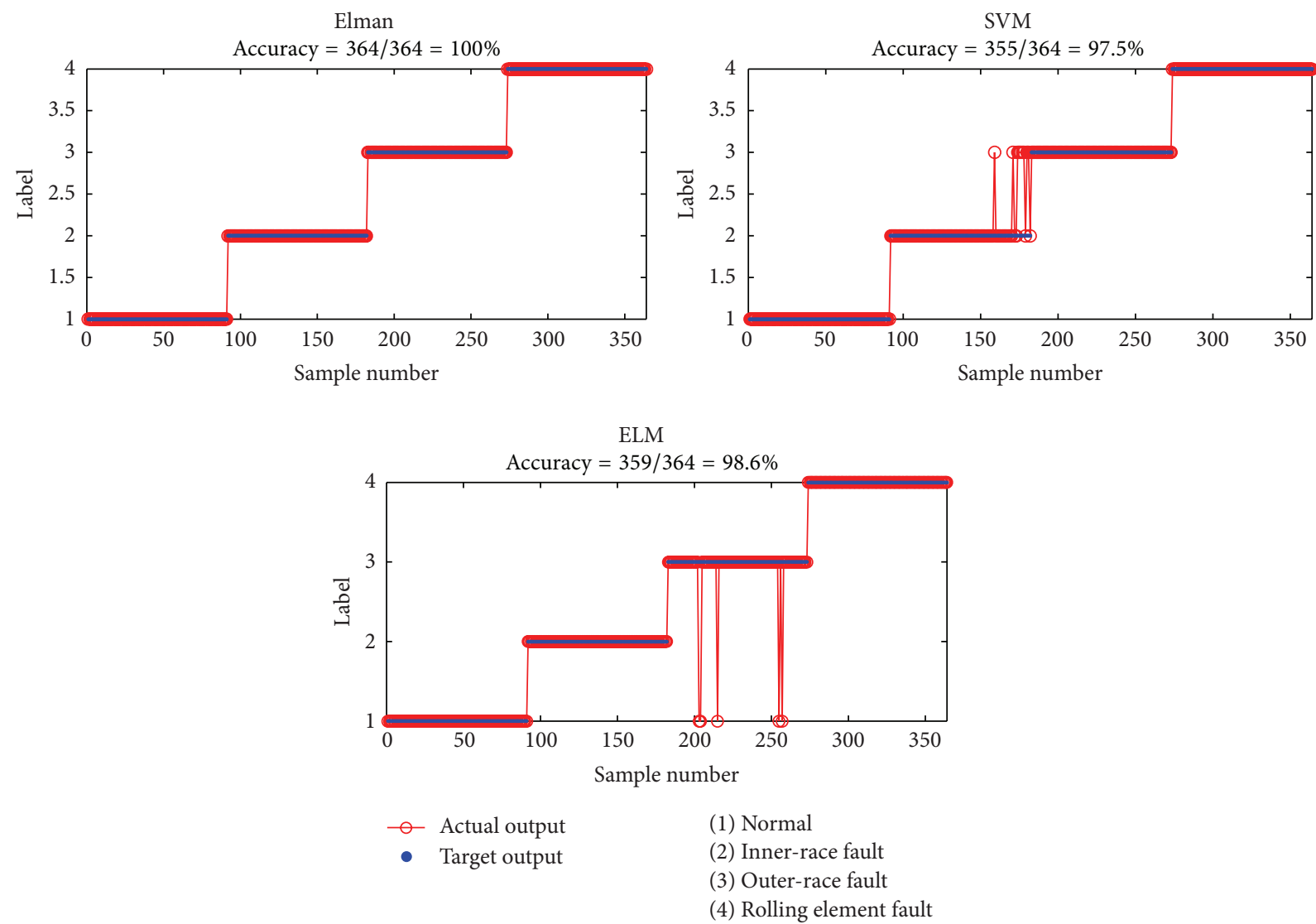

FIgURE 8: Classification performance of different classification techniques.

TABLE 3: Elman neural network recognition results.

\begin{tabular}{|c|c|c|c|c|c|c|c|}
\hline Sequence & State & Operating condition & Target output & & Actual outpr & f network & \\
\hline 1 & Normal & $(1750 \mathrm{r} / \mathrm{min}, 2 \mathrm{HP})$ & $\left(\begin{array}{llll}1 & 0 & 0 & 0\end{array}\right)$ & 0.993781 & $3.45 E-08$ & 0.043759 & 0.001039 \\
\hline 2 & & $(1720 \mathrm{r} / \mathrm{min}, 3 \mathrm{HP})$ & $\left(\begin{array}{llll}0 & 1 & 0 & 0\end{array}\right)$ & 0.0072 & 0.9910 & 0.0072 & 0.0070 \\
\hline 3 & Inner-race fault & (1750 r/min, $2 \mathrm{HP})$ & $\left(\begin{array}{llll}0 & 1 & 0 & 0\end{array}\right)$ & 0.0067 & 0.9885 & 0.0078 & 0.0079 \\
\hline 4 & 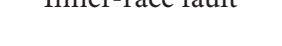 & (1772 r/min, $1 \mathrm{HP})$ & $\left(\begin{array}{llll}0 & 1 & 0 & 0\end{array}\right)$ & $2.94 E-06$ & 0.999569 & $5.87 E-05$ & 0.078958 \\
\hline 5 & & (1797 r/min, $0 \mathrm{HP})$ & $\left(\begin{array}{llll}0 & 1 & 0 & 0\end{array}\right)$ & 0.0067 & 0.9883 & 0.0078 & 0.0080 \\
\hline 6 & & $(1720 \mathrm{r} / \mathrm{min}, 3 \mathrm{HP})$ & $\left(\begin{array}{llll}0 & 0 & 1 & 0\end{array}\right)$ & 0.000413 & 0.100543 & 0.990864 & $6 E-11$ \\
\hline 7 & Outer-race & (1750 r/min, $2 \mathrm{HP})$ & $\left(\begin{array}{llll}0 & 0 & 1 & 0\end{array}\right)$ & $7.241 e-05$ & 0.0155 & 0.9845 & 0.0128 \\
\hline 8 & & (1772 r/min, $1 \mathrm{HP})$ & $\left(\begin{array}{llll}0 & 0 & 1 & 0\end{array}\right)$ & 0.000264 & 0.007905 & 0.997584 & 0.000573 \\
\hline 9 & & (1797 r/min, $0 \mathrm{HP})$ & $\left(\begin{array}{llll}0 & 0 & 1 & 0\end{array}\right)$ & $5.880 e-05$ & 0.0109 & 0.9900 & 0.0112 \\
\hline 10 & & $(1720 \mathrm{r} / \mathrm{min}, 3 \mathrm{HP})$ & $\left(\begin{array}{llll}0 & 0 & 0 & 1\end{array}\right)$ & 0.0051 & 0.000405 & 0.0109 & 0.9866 \\
\hline 11 & Rolling element fault & $(1750 \mathrm{r} / \mathrm{min}, 2 \mathrm{HP})$ & $\left(\begin{array}{llll}0 & 0 & 0 & 1\end{array}\right)$ & 0.0051 & 0.000236 & 0.0117 & 0.9898 \\
\hline 12 & 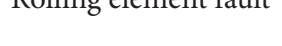 & (1772 r/min, $1 \mathrm{HP})$ & $\left(\begin{array}{llll}0 & 0 & 0 & 1\end{array}\right)$ & 0.0064 & 0.000245 & 0.0100 & 0.9899 \\
\hline 13 & & (1797 r/min, $0 \mathrm{HP})$ & $\left(\begin{array}{llll}0 & 0 & 0 & 1\end{array}\right)$ & 0.0100 & 0.000583 & 0.0072 & 0.9815 \\
\hline
\end{tabular}

and an inner-race fault was injected at $0.5 \mathrm{~s}$. The initial motor load was $3 \mathrm{HP}$, which changed $0 \mathrm{HP}$ at $0.3 \mathrm{~s}$ and $1 \mathrm{HP}$ at $0.7 \mathrm{~s}$. The instantaneous amplitude matrices are analyzed by conducting SVD for every $0.05 \mathrm{~s}$. Figure 10 shows the corresponding instantaneous amplitude and singular value $\sigma_{n}(t)$. The function $\sigma_{n}(t)$ remains relatively stable in one state. With the alteration of the motor load, the change of $\sigma_{n}(t)$ is not obvious. After an unexpected fault is injected, the value of $\sigma_{1}(t)$ changes rapidly. Moreover, even when the motor load changes to 1HP, the fault identification could still be achieved by observing the value of $\sigma_{n}(t)$.

After the detection of a burst fault, this method is used to measure the fault severity under time-varying condition in advance, which is illustrated in Figure 11. To realize fault injection of different severities, grooves with different diameters were engraved on different positions of bearings 

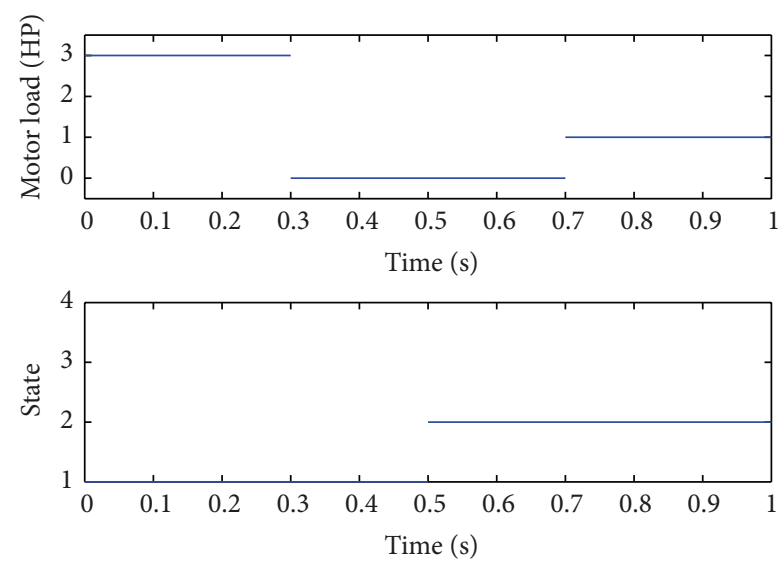

(1) Normal

(3) Outer-race fault

(2) Inner-race fault

(4) Rolling element fault

FIGURE 9: Diagram of the time-varying condition.
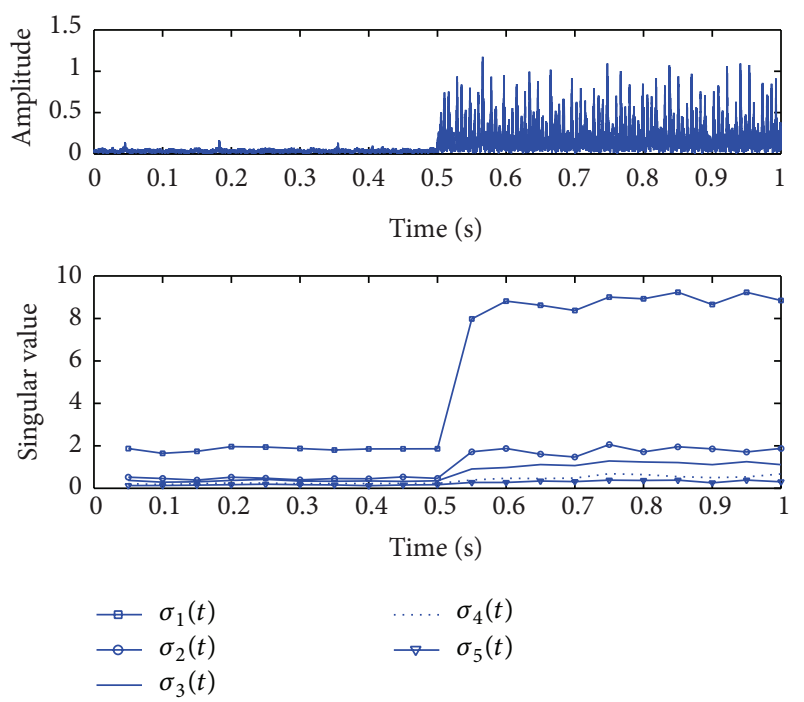

FIGURE 10: Diagram of instantaneous amplitude and singular value $\sigma_{n}(t)$ corresponding to Figure 9.
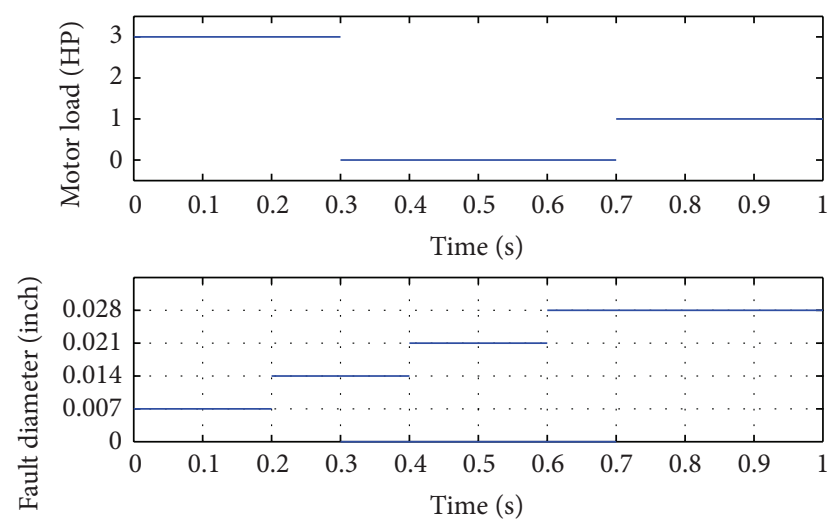

FIGURE 11: Diagram of the time-varying fault diameter.
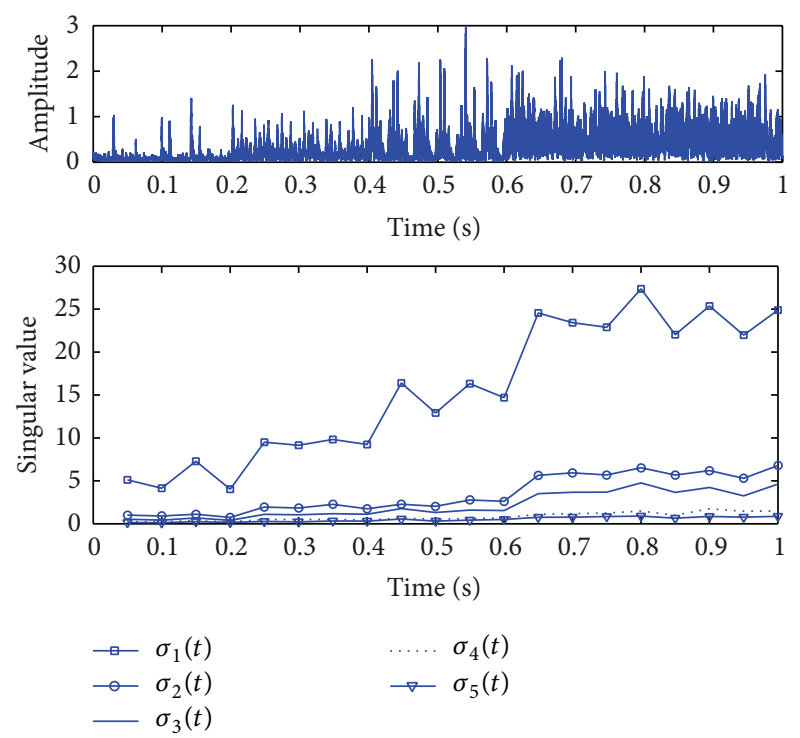

FIGURE 12: Diagram of instantaneous amplitude and singular value $\sigma_{n}(t)$ corresponding to Figure 11.

(inner race, outer race, and rolling element), and fault diameter is used to represent fault severity. Here, the data is acquired by connecting data segments under different working conditions and different fault severities. The unit of fault diameter in this paper is inch. The initial motor load was $3 \mathrm{HP}$, which changed to $0 \mathrm{HP}$ at $0.3 \mathrm{~s}$ and $1 \mathrm{HP}$ at $0.7 \mathrm{~s}$. In addition, the initial fault diameter of inner-race fault was 0.007 inches, which changed to 0.014 inches at $0.2 \mathrm{~s}$, 0.021 inches at $0.4 \mathrm{~s}$, and 0.028 inches at $0.6 \mathrm{~s}$. Similarly, the instantaneous amplitude matrices are analyzed by conducting SVD for every $0.05 \mathrm{~s}$. Figure 12 shows the corresponding instantaneous amplitude and singular value $\sigma_{n}(t)$, from which we can figure that the function $\sigma_{n}(t)$ remains relatively stable under a fault severity. With the alteration of the motor load, the change of $\sigma_{n}(t)$ is not obvious. The function $\sigma_{n}(t)$ tends to increase simultaneously with the fault severity. To this end, after an early slight fault is diagnosed under variable working conditions, the proposed method can be employed to observe whether the fault severity is deepened in advance.

\section{Conclusion}

Rolling bearing fault diagnosis under time-varying working condition has always been a thorny issue in the field of bearing fault diagnosis. This paper presents a new fault diagnosis method combining HHT, SVD, and Elman neural network. HHT can generate the time domain signal and the frequency domain signal at the same time; thus, it supplies abundant information for the fault diagnosis under variable conditions. Since the time-frequency feature vector generated by the HHT is indeed redundant, the singular value decomposition is proposed in this paper for reducing dimensionality and obtaining feature insensitive to working condition variation, which is beneficial to enhance the classification accuracy under variable working conditions. 
First, the IMFs were obtained by applying EMD. Second, the instantaneous amplitude matrices were calculated by applying Hilbert transform to each IMF. The matrix was then decomposed by SVD. This method finds out the singular value commonality for different operating conditions, which achieves rolling bearing fault diagnosis under time-varying conditions. Finally, the Elman neural network was used for fault identification and classification according to the extracted feature vector. The experimental results demonstrated the effectiveness of the singular value for different operating conditions.

Taking the existing EMD-SVD and WPT-PCA for comparison, we demonstrated the robustness of the proposed feature extraction based on HHT-SVD. Finally, by comparison to SVM and ELM, the effectiveness of the proposed method using the Elman neural network for classification was further verified. Based on the preceding discussion, the superiority of the proposed method in handling fault diagnosis under the time-varying condition can be verified. Without the need for human intervention and additional cost, this approach is an excellent automatic method that can be promoted for fault diagnosis under variable conditions.

\section{Conflict of Interests}

The authors declare that there is no conflict of interests regarding the publication of this paper.

\section{Acknowledgments}

This research is supported by the National Natural Science Foundation of China (Grant nos. 51105019, 61074083, and 50705005) and the Technology Foundation Program of National Defense (Grant no. Z132013B002).

\section{References}

[1] B. Allotta, R. Papi, L. Pugi, P. Toni, and A. G. Violi, "Experimental campaign on a servo-actuated pantograph," in Proceedings of the IEEE/ASME International Conference on Advanced Intelligent Mechatronics, vol. 1, pp. 237-242, Como, Italy, July 2001.

[2] Y. Shao, L. Ge, and J. Fang, "Fault diagnosis system based on smart bearing," in Proceedings of the International Conference on Control, Automation and Systems (ICCAS '08), pp. 1084-1089, July 2008.

[3] Y. Yang, H. Wang, J. Cheng, and K. Zhang, "A fault diagnosis approach for roller bearing based on VPMCD under variable speed condition," Measurement, vol. 46, no. 8, pp. 2306-2312, 2013.

[4] Q. He, Y. Liu, Q. Long, and J. Wang, “Time-frequency manifold as a signature for machine health diagnosis," IEEE Transactions on Instrumentation and Measurement, vol. 61, no. 5, pp. 12181230, 2012.

[5] X. G. Tang, Y. Guo, and Y. C. Zheng, "Application of rolling element bearing envelope analysis based on short time Fourier transition and independent components analysis," Journal of Mechanical Strength, vol. 34, no. 1, pp. 1-3, 2012.

[6] L. Shi, Y. Zhang, and W. Mi, "Application of Wigner-Villedistribution-based spectral kurtosis algorithm to fault diagnosis of rolling bearing," Journal of Vibration, Measurement and Diagnosis, vol. 31, no. 1, pp. 27-31, 2011.

[7] P. K. Kankar, S. C. Sharma, and S. P. Harsha, "Rolling element bearing fault diagnosis using wavelet transform," Neurocomputing, vol. 74, no. 10, pp. 1638-1645, 2011.

[8] Z. Feng, M. Liang, and F. Chu, "Recent advances in timefrequency analysis methods for machinery fault diagnosis: a review with application examples," Mechanical Systems and Signal Processing, vol. 38, no. 1, pp. 165-205, 2013.

[9] N. E. Huang and S. S. Shen, Hilbert-Huang Transform and Its Applications, World Scientific, Singapore, 2005.

[10] N. E. Huang, Z. Shen, S. R. Long et al., "The empirical mode decomposition and the Hilbert spectrum for nonlinear and non-stationary time series analysis," Proceedings of the Royal Society of London Series A: Mathematical, Physical and Engineering Sciences, vol. 454, no. 1971, pp. 903-995, 1998.

[11] M. G. Safonov, "Stability margins of diagonally perturbed multivariable feedback systems," IEE Proceedings D Control Theory and Applications, vol. 129, no. 6, pp. 251-256, 1982.

[12] J. Yang, Y. Zhang, and Y. Zhu, "Intelligent fault diagnosis of rolling element bearing based on SVMs and fractal dimension," Mechanical Systems and Signal Processing, vol. 21, no. 5, pp. 2012-2024, 2007.

[13] L. Lei and Q. Zhang, "Relevance Vector Machine based bearing fault diagnosis," in Proceedings of the IEEE International Conference on Machine Learning and Cybernetics, pp. 3492-3496, Dalian, China, August 2006.

[14] G. B. Huang, Q. Y. Zhu, and C. K. Siew, "Extreme learning machine: a new learning scheme of feedforward neural networks," in Proceedings of the IEEE International Joint Conference on Neural Networks, pp. 985-990, July 2004.

[15] H. Li and Y. Zhang, "Bearing localized fault detection based on Hilbert-Huang transformation," in Proceedings of the 4th International Conference on Fuzzy Systems and Knowledge Discovery (FSKD '07), pp. 138-142, August 2007.

[16] Y. Yang, D. Yu, and J. Cheng, "A fault diagnosis approach for roller bearing based on IMF envelope spectrum and SVM," Measurement, vol. 40, no. 9-10, pp. 943-950, 2007.

[17] R. Zhan and H. Wu, "Feature extraction \& application of engineering non-stationary signals based on EMD-AR model and SVD," in Proceedings of the IEEE International Conference on Computer Design and Applications (ICCDA '10), pp. V4-528V4-532, June 2010.

[18] Z. Qi, M. Liu, and H. Wang, "Process modeling method based on an improved Elman Neural Network," in Proceedings of the 7th World Congress on Intelligent Control and Automation (WCICA '08), pp. 8188-8192, Chongqing, China, June 2008.

[19] J. Cheng, D. Yu, J. Tang, and Y. Yang, "Application of SVM and SVD technique based on EMD to the fault diagnosis of the rotating machinery," Shock and Vibration, vol. 16, no. 1, pp. 8998, 2009.

[20] Z. Zhu and Y. Sun, "Application of EMD and SVD in fault identification," in Proceedings of the 8th International Power Engineering Conference (IPEC '07), pp. 1247-1250, April 2007.

[21] R. Shao, W. Hu, Y. Wang, and X. Qi, “The fault feature extraction and classification of gear using principal component analysis and kernel principal component analysis based on the wavelet packet transform," Measurement, vol. 54, pp. 118-132, 2014. 


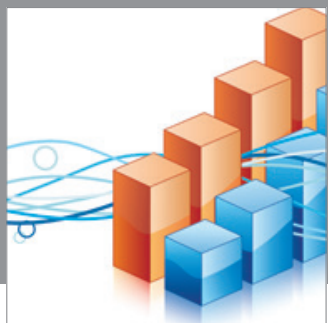

Advances in

Operations Research

mansans

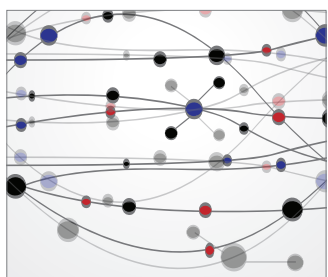

The Scientific World Journal
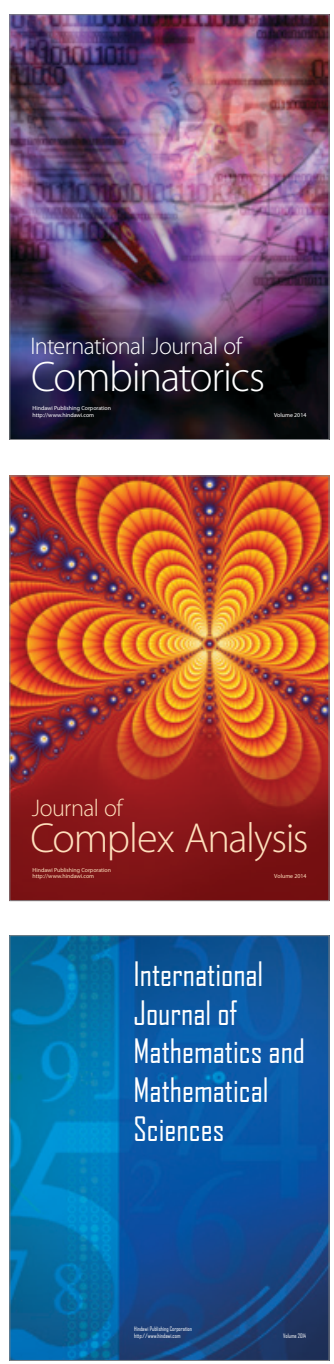
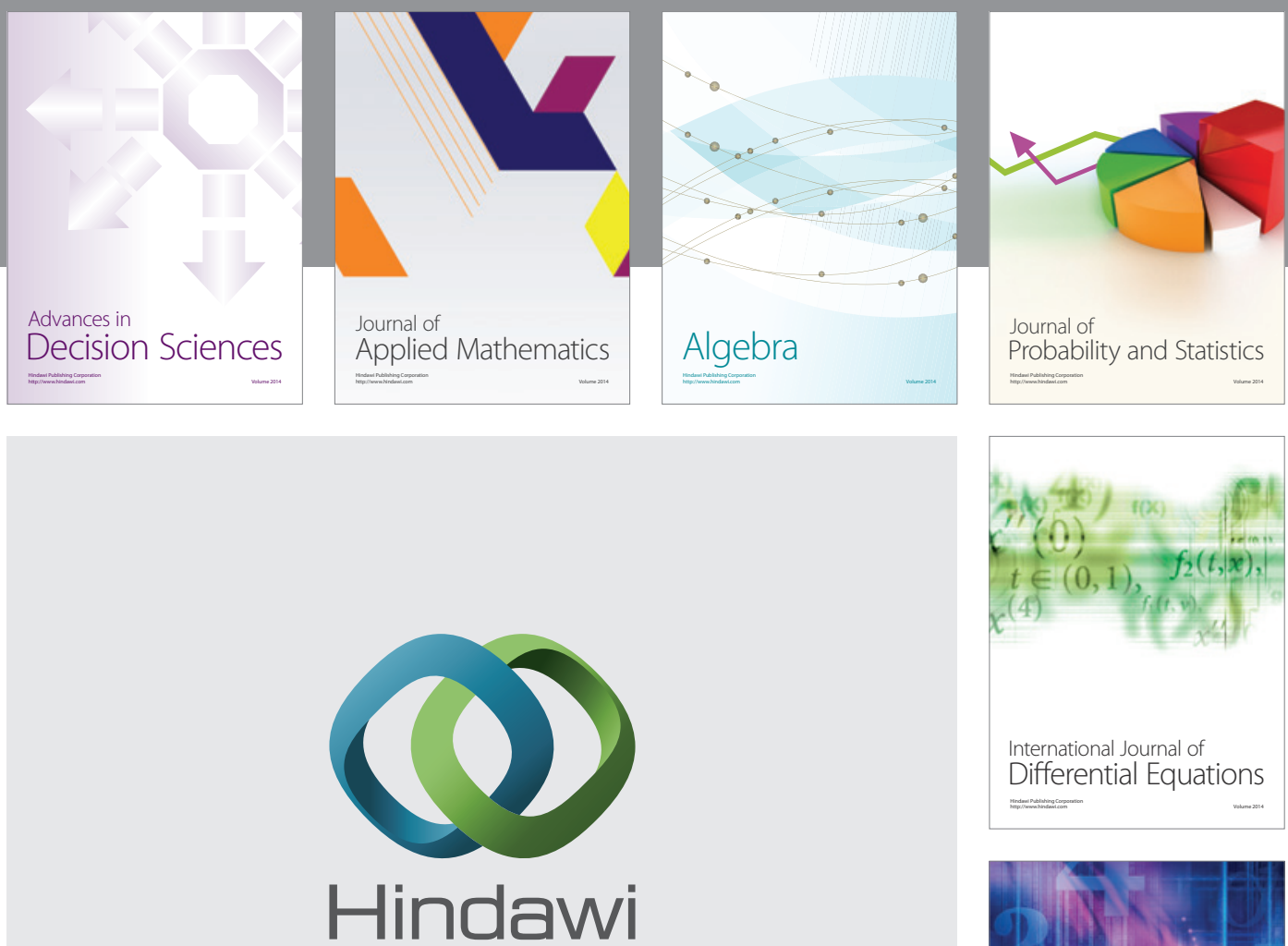

Submit your manuscripts at http://www.hindawi.com
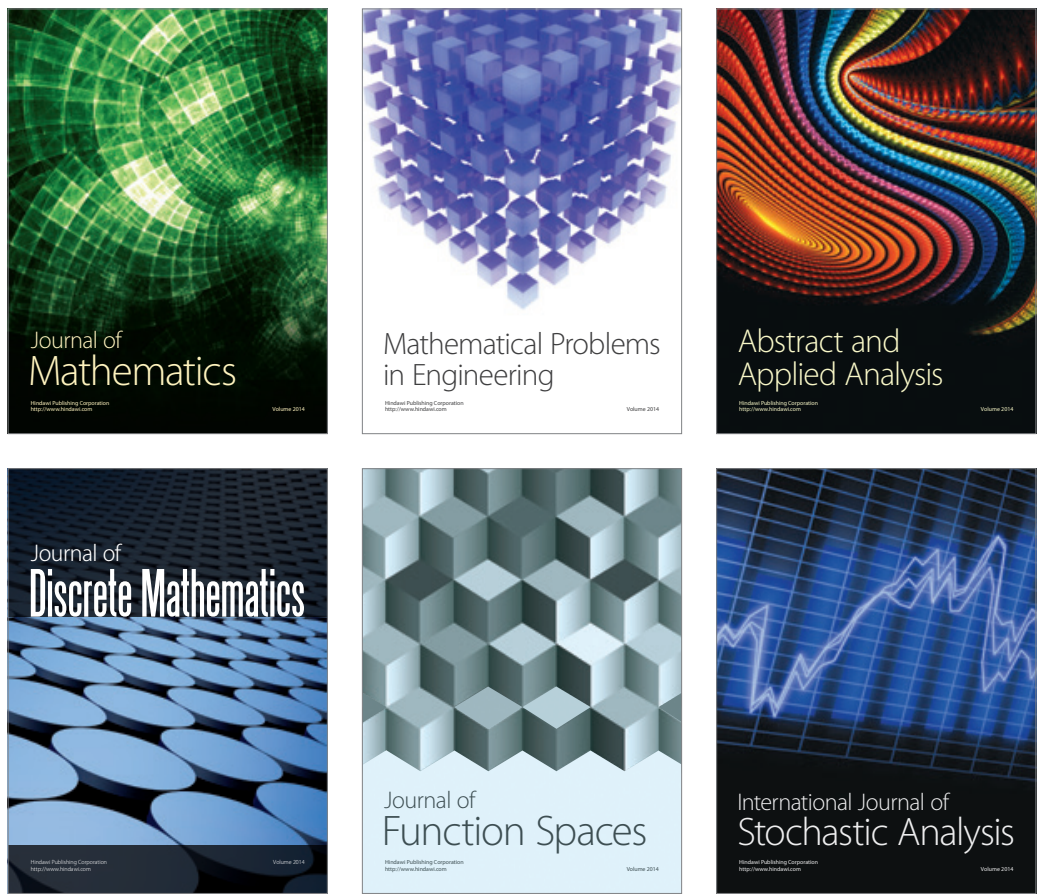

Journal of

Function Spaces

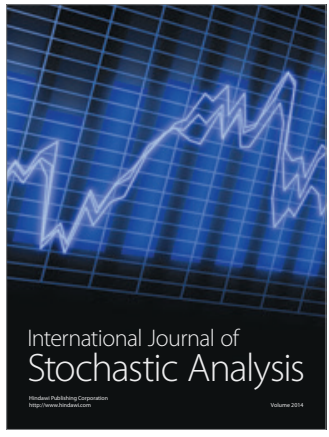

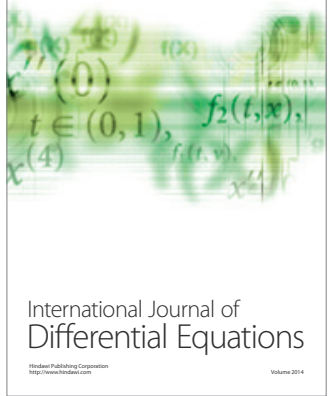
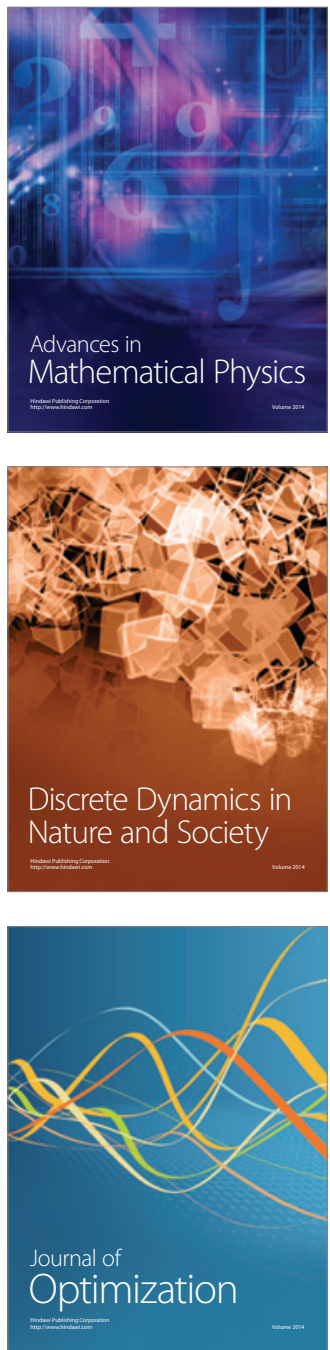